\title{
Design Optimization of Miniature Magnetorheological Valves with Self-Sensing Capabilities Used for a Wearable Medical Application
}

\author{
Sofia Lydia Ntella ${ }^{1}$, Minh-Trung Duong ${ }^{1}$, Yoan Civet ${ }^{1}$, Zoltan Pataky ${ }^{2}$ and Yves Perriard ${ }^{1}$
}

\begin{abstract}
Magnetorheological (MR) fluids are materials characterized as "intelligent" since their rheological properties may be controlled by the excitation of an external magnetic field. The application of the latter brings the fluid from a liquid to a semi-solid state. Due to their properties, these materials are widely used, among others, in biomedical applications. In the present work, multiple MR valves are incorporated as pressure limiters in a smart biomedical insole for diabetic patients. The diabetics are affected by high plantar pressures that cause ulceration and frequently, as a consequence of ulceration, lower extremity amputation. This novel insole aims at the active plantar pressure offloading. Thus, considering the small size of the insole, the number of the valves, as well as the high plantar pressures that reach up to $1 \mathrm{MPa}$ on diabetic feet, a study is performed on optimizing the design of the MR valve, exploiting the Design of Experiments (DoE) techniques. Moreover, a testbench is presented for the examination of pressure self-sensing capabilities of the valves, with a future goal to integrate them in the design optimization and acquire a sensorless system.
\end{abstract}

Index Terms-MR pressure limiters, design optimization, DoE, sensorless system, smart footwear for diabetics

\section{INTRODUCTION}

MR fluids are smart materials that consist of ferromagnetic particles dispersed in a non-magnetic liquid carrier medium [1]. When an external magnetic field is applied on them, the ferromagnetic particles form chain-like structures in parallel with the lines of the magnetic field, as in Fig. 1. Due to the formation of the structures, a reversible change of the fluid's viscosity occurs and the MR fluid passes from a liquid to a semi-solid state [2].

The MR valve, as the one shown in Fig. 2, is the fundamental component of a MR device [3]. It exploits the properties of viscosity variability of MR fluids for different applications, such as shock absorbers [4], [5], actuators [6] and dampers [7]. Such a valve consists of a ferromagnetic core, a coil for the excitation of the magnetic field and an air gap through which the MR fluid flows. The regions of the gap, where the flow is perpendicular to the magnetic field are called active regions. This place is where the MR effect occurs. The MR valves may be found in different configurations such as annular, radial or annular-radial [8], according to the arrangement of the gap and, thus, the fluid flow.

\footnotetext{
${ }^{1}$ Integrated Actuators Laboratory (LAI), École Polytechnique Fédérale de Lausanne (EPFL), Neuchâtel, Switzerland sofia.ntella@epfl.ch

${ }^{2}$ Division of Endocrinology, Diabetology, Nutrition and Therapeutic Education, Hôpitaux Universitaires de Genève
}

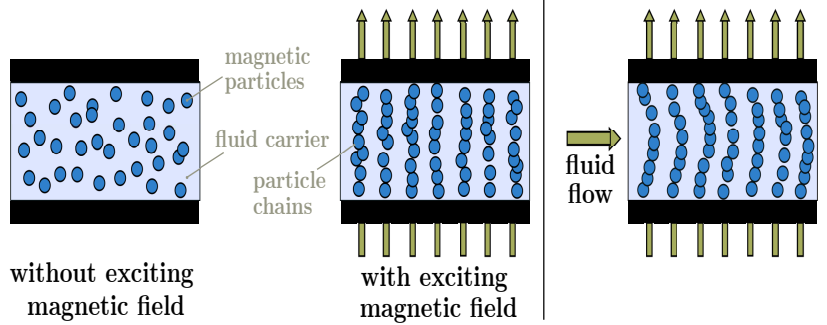

Fig. 1: Ferromagnetic particles distribution of a magnetorheological fluid without and with exciting magnetic field and chain stretching due to fluid flow.

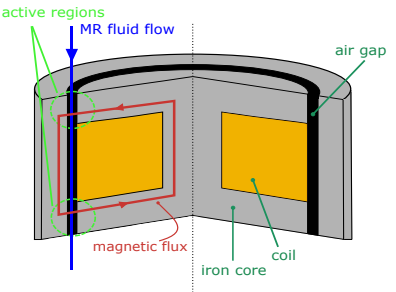

Fig. 2: Annular magnetorheological valve structure, with a coil winded on the inner ferromagnetic core.

In this paper, the MR valves under examination, are used as pressure limiters in a wearable medical application for diabetic patients. These patients suffer from a combination of high plantar pressures (PP), that cause ulceration, and peripheral neuropathy, which leads to the loss of sensation in the lower limbs [9]. Several solutions, such as orthopaedic and custom-made insoles are proposed nowadays for the prevention of ulceration [10]. However, they are cumbersome and implement only passive offloading. Our aim is to create for the first time a smart wearable device in an insole form, that performs continuous monitoring of PP distribution and automatic offloading of the regions that present high pressure values.

For this reason, the insole will be discretized in pressure measurement regions and each region will correspond to one MR module, as the one depicted on Fig. 3. Each module consists of a MR valve, and two chambers, one on top and on one the bottom of the valve, that contain the MR fluid. A membrane pushes back the fluid from the bottom to the top chamber and the cushion on the top of the whole structure allows deformation. Finally, a pressure sensor above the cushion monitors the pressure in each plantar region. The 
module functions as the pressure limiter and deforms in case of high pressure values in order to achieve offloading.

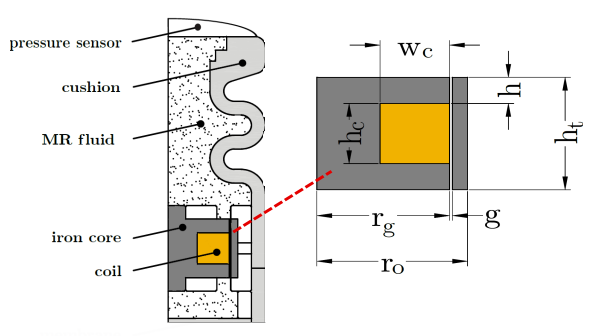

Fig. 3: 2D axis-symmetric cross-section of MR module, used in the amputation prevention smart footwear, and MR vavle dimensions.

Previous studies [11] successfully designed a working MR valve for the insole. However, they did not obtain the optimized model considering all the different affecting parameters. More specifically, taking into consideration the final goal, which is a wearable solution, the large number of valves per insole, as well as the high loads that must be sustained due to the high PP on the diabetic feet, the need for optimization seems crucial. In the current work, we optimize the design of the valve in terms of volume, pressure drop and power consumption using DoE techniques and we achieve to acquire a miniature design that satisfies the needs of our system in the optimum way.

Other studies [12], [13], [14] have also revealed selfsensing properties for the MR valves, observing an induced voltage on the coil. Nevertheless, either they only focus on displacement and velocity sensing or the have not managed to quantify the self-sensing phenomena with regards to pressure variations. The testbench designed and assembled to investigate these properties is described in this paper. Its target is to characterize the induced signal and relate it to pressure changes. Such a work will facilitate the utmost aim of our research, which is the elimination of pressure sensors from the MR module and the accomplishment of a sensorless system.

\section{MR VALVE SELF-SENSING PROPERTIES IDENTIFICATION}

The initial idea for our system design relies on the placement of a pressure sensor on top of each MR module structure, as described previously. The sensor is supposed to measure the PP of the corresponding plantar region. The measurement will be the input for the micro-controller that drives the system. The latter will either offload or sustain the load on this part of the plantar surface. The characterization of the induced voltage observed on the valve's coil in case of pressure and flow variations will provide the possibility of a novel sensorless device, freed from the need of pressure sensors. Considering the fact that the insole area will consist of 30 to $50 \mathrm{MR}$ valves, depending on the patient's foot size, we hope to achieve a significant decrease of the the cost of the wearable with the sensorless system and convert it to a more appealing solution to diabetic patients.

The induced voltage may be explained by the variation of reluctance in the active regions of the valve's gap. More precisely, the reluctance $\mathcal{R}_{M R}$ of the MR fluid in the gap can be described by the following equation [15]:

$$
\mathcal{R}_{M R}=\frac{\ln \left(1+\frac{g}{r_{g}}\right)}{\pi h \mu_{0} \mu_{M R}}
$$

where $g$ is the valve's gap length, $r_{g}$ is the inner core radius of the valve, $\mathrm{h}$ is the active regions height, $\mu_{0}$ is the free space permeability and $\mu_{M R}$ is the relative permeability of the MR fluid. Considering that $r_{g}>>g$, the above equation is simplified as follows:

$$
\mathcal{R}_{M R}=\frac{g(t)}{\pi r_{g} h \mu_{0} \mu_{M R}}
$$

Here, $g(t)$ can be considered as the magnetic particles chain length, which is the only variable in time parameter, assuming that the concentration of the particles in the gap is constant. Additionally, if $N$ is the number of turns and $i$ is the current on the valve's coil, the magnetic flux $\Phi$ in the gap can be expressed as:

$$
\Phi(t)=\frac{N \cdot i}{\mathcal{R}_{M R}}
$$

It is, thus, obvious that the reluctance of the gap, and as a result the magnetic flux is dependent on the particle chain length, which stretches when a pressure is applied on the fluid. This explains the appearance of an induced voltage, which may depend on the applied pressure on the chain.

The testbench depicted on Fig. 4 was assembled in order to investigate the induced voltage on the coil of the MR valve. It consists of the following parts: 1) The MR valve with two coils winded around the inner magnetic core (Fig. 4). A current of minimum $50 \mathrm{~mA}$ is provided to the inner coil (exciting coil), with the aim to create a magnetic field and, as a result, some initial formations of ferromagnetic chainlike structures in the MR fluid. The existence of induced voltage is due to the reluctance variation of the MR fluid in the active regions, since the flow and pressure variations cause the deformation of the MR fluid chains. The resistance of the inner coil is $R_{i}=0.372 \Omega$ and the number of turns is $N_{i}=10$. The outer coil is the sensing coil, with $R_{o}=0.637$ $\Omega$ and $N_{o}=40$ turns. In this work, the number of turns of the sensing coil is increased compared to previous ones [12], in order to increase the induced signal. 2) The fluid chambers, in the middle of which, the valve is placed. 3) The pneumatic cylinder piston SMC CDQ2A32TF-50DZ that forces the fluid from one chamber to the other, through the MR valve. 4) The LK-G150 laser displacement sensor with LK-G3001PV all-in-one-controller to measure the displacement and consequently the velocity of the piston rod. By multiplying this velocity with the cross-section area $A=12.56 \mathrm{~cm}^{2}$ of the piston rod, we acquire the fluid's flow rate every time. 5) A 3/5 pneumatic valve that supplies the cylinder with compressed air. 6) Two MS5803-14BA pressure sensors, 


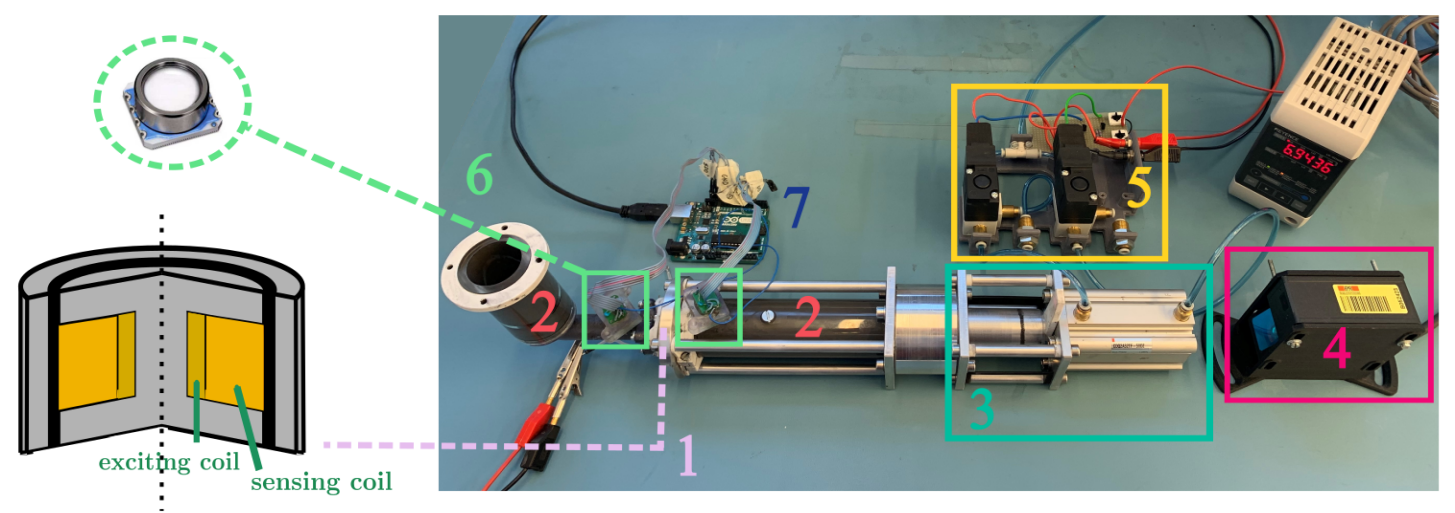

Fig. 4: Testbench set-up, with a 2-coil annular MR valve for investigating the valve's pressure self-sensing possibilities.

one for each chamber, connected to an Arduino Uno device through $\mathrm{I}^{2} \mathrm{C}$ communication. 7) An electronic circuit with an AD708JN instrumentation amplifier from Analog Devices to amplify the sub-millivolt induced voltage. The latter is read by Arduino analog pins. In addition, enamelled copper wire by Elektrisola with a diameter of $0.224 \mathrm{~mm}$ of bare copper, thinner than in previous experiments [12] is used in order to avoid scratches and short circuits and reassure the validity of the results. The MR fluid used in this experiments is the MRF132DG by Lord Corporation.

In future steps we aim at using the experimental trials to characterize the induced voltage and correlate it with the pressure variations. When this is accomplished, the experiments may be repeated for different MR valve prototypes, and include these results as a factor of the DoE optimization procedure.

\section{DESIGN OPTIMIZATION OF MR VALVE}

\section{A. Problem Parameters Definition}

The magnetic flux in any region of the MR valve depends on the geometric parameters of the valve as well as on the type of the MR fluid used for the application. In Fig. 3 the different dimensions of the valve, used in the optimization process, were defined. More precisely, $r_{o}$ and $r_{g}$ are the outer and inner magnetic core radius respectively, $g$ is the gap length, $h_{t}$ is the valve's total height, $h$ is iron teeth's height, while $h_{c}$ and $w_{c}$ are the coil's height and width respectively.

The objectives of the optimization approach are: the minimization of the valve's volume $V$ and power consumption $P$ and the maximization of flow rate $Q$ and pressure drop $\Delta \mathrm{P}$ in the valve, with a minimum limit of $700 \mathrm{kPa}$. Regarding the pressure drop limit, plantar pressure distribution studies on diabetic [16] and healthy feet [17] have proven that for the second case the PP ranges between 350 and $400 \mathrm{kPa}$ in the forefoot and heel areas, while for the first case it may range from $700 \mathrm{kPa}$ up to $1 \mathrm{Mpa}$. Therefore, it is crucial for the MR module to be able to sustain $350-400 \mathrm{kPa}$ on the healthy areas. Considering, also, the safety range for the design and manufacturing of the valve, we increase the margin of sustainable load from $400 \mathrm{kPa}$ to $700 \mathrm{kPa}$, in order to achieve higher tolerance.

\section{B. Fractional Factorial Design}

The fractional factorial design is a widely known and commonly used method for the identification of critical effects and factors on the response of a system [18]. It facilitates the decrease of the size of an experiment, in case a high number of factors are included, allowing, in parallel a trade-off for the loss of significant information due to the reduction of the number of experimental trials.

Table I includes the factors (dimensions of the valve, ratios of dimensions and current density of the coil) and their corresponding levels chosen for the current factorial design. Considering the fact that our experiment involves 7 different factors, the choice of a fractional and not full factorial design appears to be the most efficient.

TABLE I: Definition of Factors \& Levels for the Fractional Factorial Design

\begin{tabular}{|c|c|c|c|}
\hline & Factor & Low & High \\
\hline 1 & Current density J $\left(\mathrm{A} / \mathrm{mm}^{2}\right)$ & 9.0 & 15.0 \\
\hline 2 & Outer core radius $\mathrm{r}_{\mathrm{o}}(\mathrm{mm})$ & 4.0 & 6.0 \\
\hline 3 & Total valve's height $\mathrm{h}_{\mathrm{t}}(\mathrm{mm})$ & 4.0 & 7.0 \\
\hline 4 & Air gap g $(\mathrm{mm})$ & 0.15 & 0.3 \\
\hline 5 & $\mathrm{~h}_{\mathrm{c}} / \mathrm{h}_{\mathrm{t}}$ & 0.4 & 0.6 \\
\hline 6 & $\mathrm{r}_{\mathrm{g}} / \mathrm{r}_{\mathrm{o}}$ & 0.6 & 0.8 \\
\hline 7 & $\mathrm{w}_{\mathrm{c}} / \mathrm{r}_{\mathrm{g}}$ & 0.5 & 0.7 \\
\hline
\end{tabular}

Minitab 19 was used for the DoE optimization. Firstly, the different combinations of factors and levels were defined. The levels of current density were chosen higher than in the existing prototype (Table II), considering that in smaller volume, higher currents are necessary for load sustainment. The levels of dimensions were chosen so that the total volume, diameter and height of the valve satisfy the requirements for miniaturization. For each combination of factors and levels, a simulation of the magnetic field distribution in the MR valve gap was performed, using Flux 2018.1.

The magnetic flux density $B$, that was obtained from the simulation, was used for the calculation of the magnetic 
field intensity $H$ and yield stress $\tau_{y}$ of the MR fluid. More specifically, the $B-H$ and $\tau_{y}-H$ curves of MRF132DG, as in Fig. 5, were used. These graphs derive from the MR fluid datasheet by Lord Corporation. Curve fitting interpolation was used to acquire the two following equations:

$$
\begin{gathered}
H=-102.64 B^{4}+324.64 B^{3} \\
-91.47 B^{2}+180.01 B-0.17 \\
\tau_{y}=1.39 \times 10^{-8} H^{4}-7.41 \times 10^{-6} H^{3} \\
+5.06 \times 10^{-4} H^{2}+0.31 H-0.40
\end{gathered}
$$

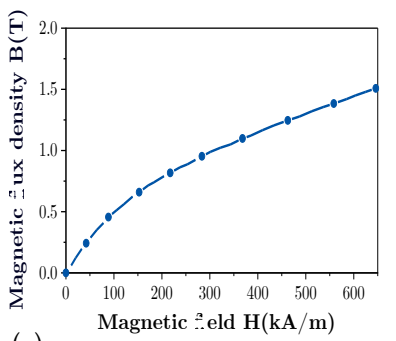

(a)

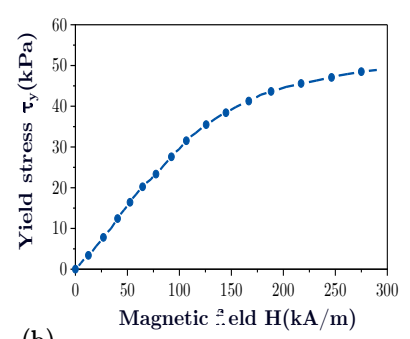

(b)
Fig. 5: MRF132DG fluid (a) $B-H$ curve and (b) $\tau_{\mathrm{y}}-H$ curve.

Initially, the magnetic field intensity $H$ was calculated using the Eq. 4 and then the yield stress was calculated using the previously obtained magnetic field intensity $H$ and the Eq. 5. The calculation of the pressure drop and dynamic range was achieved according to the following equations [19]:

$$
\begin{gathered}
\Delta P=\Delta P_{\tau}+\Delta P_{\eta}=\frac{c \tau_{y}(H) 2 h}{g}+\frac{6 \eta Q 2 h}{g^{3} \pi r_{g}} \\
D=\frac{\Delta P_{\tau}}{\Delta P_{\eta}}=5
\end{gathered}
$$

where $\Delta P$ is the total pressure drop, $\Delta P_{\tau}$ is the yield stress dependent pressure drop, $\Delta P_{\eta}$ is the viscosity dependent pressure drop and $D$ is the dynamic range, defined as the ratio between the two previous pressure drops, $h$ is the teeth's height, $\eta$ is the MR fluid's viscosity and $Q$ is the fluid's flow rate. $D$ is a figure of merit that defines the valve's operation range, from a free flow to a no flow condition. The parameter c ranges from a minimum value 2 for dynamic range $D$ less than 1 , to a maximum value 3 , for dynamic range $D$ greater than 100. In this case, the dynamic range is chosen to be 5 [11], so c value was approximated to 2.3 [20].

By using the Eq. 4 and and the value 5 for the dynamic range, we calculated the flow rate. Finally, the volume of the MR valve was calculated according to the following equation:

$$
V=\pi r_{o}^{2} h_{t}
$$

where $\mathrm{V}$ is the total volume of the magetorheological valve, including both the inner and outer ferromagnetic core, the coil volume and the gap volume.

The pareto charts of Fig. 6 indicate the standardized effect of the various factors mentioned before on pressure drop
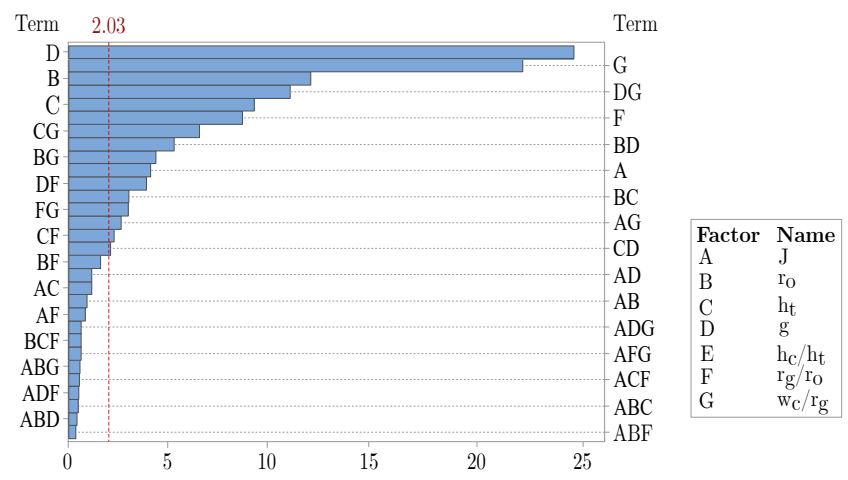

(a) Standardized effects on pressure drop.

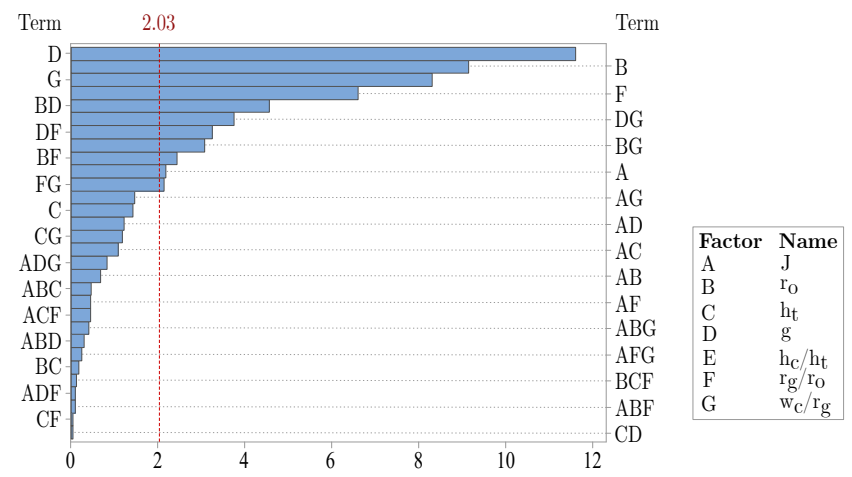

(b) Standardized effects on flow rate.

Fig. 6: Pareto charts.

$\Delta P$ and flow rate $Q$ as response respectively, with factor D presenting the highest effect. Minitab has automatically eliminated the factor $h_{c} / h_{t}$ from the pareto chart, showing that it is the least important of all factors. Thus, we also eliminate it from our design and simplify the next steps of optimization.

\section{Response Surface Method}

The fractional factorial design implemented the screening of the significant factors from the less important ones. The second step in the optimization process is the use of the Response Surface Methodology (RSM) with the aim to obtain the optimized level of factors for the MR valve. RSM is a sequential process, where initially an approximation of the relationship between the factors and the response is defined. In the end of the process the response surface and the optimized factor levels and response are acquired [21].

In this case, considering the size of the experiment, we, initially, reduce the number of factors from seven to six, setting the factor $h_{c} / h_{t}$ to 0.5 . According to the previous results, the factor $h_{c} / h_{t}$ has no impact on the responses, so we randomly pick the value 0.5 from the initially defined range. Additionally, a further decrease to five factors is accomplished, by defining the valve's outer core radius to the constant value $r_{o}=5 \mathrm{~mm}$. This value satisfies the requirements of our wearable system for sufficient pressure sensing resolution and system complexity. More specifically, the reference value for the surface of the each PP measurement region is fixed to $1.5 \mathrm{~cm}^{2}$ in previous studies [22], 
which means a radius of $6.9 \mathrm{~mm}$ for each measurement region. We must note at this point, that in the radius of 6.9 $\mathrm{mm}$ we include not only the radius of the MR valve $(5 \mathrm{~mm})$, but also the radius of the outer plastic cover $(5.8 \mathrm{~mm})$ around the valve as well as the radius of the cushion around it (6.9 $\mathrm{mm})$.

The power consumption is calculated as $P=i^{2} R$, where $i$ is the current and $R$ the resistance of the coil. The selected coil wire diameter is of type AWG28 with diameter $D_{w}=$ $0.321 \mathrm{~mm}$, while the coil fill factor is $50 \%$.

\section{RESULTS AND DISCUSSION}

As mentioned above, the goal of this work is the definition of the optimal model of the MR valve, so as to achieve minimum volume and power consumption, as well as maximum pressure drop and flow rate. Fig. 7 presents the effects of the five factors on the objectives of the experiment, which are the pressure drop, the flow rate and the power consumption. It is clearly observed that the air gap $g$ length impacts significantly both the pressure drop and the flow rate, compared to the rest of the factors. Further, the ratio $r_{g} / r_{o}$ appears to have a strong effect on flow rate, too. Last but not least, the same ratio impacts the power consumption, together with the current density, which, as logically expected, affects the most this response.
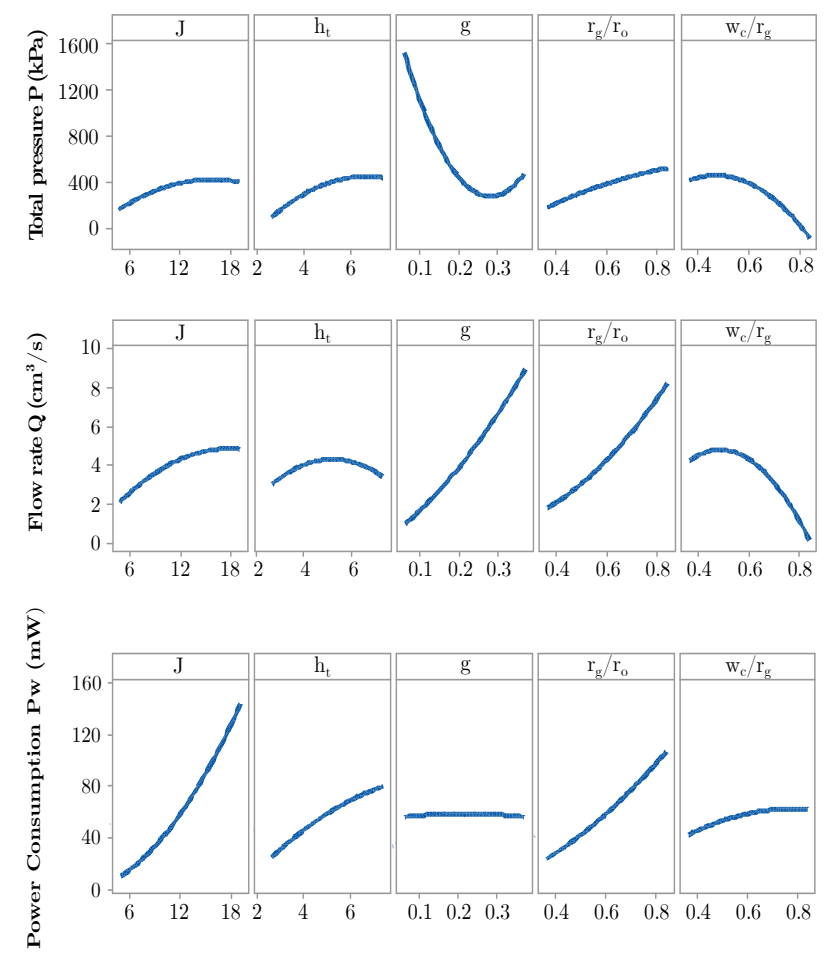

Fig. 7: Main effects of experiment factors on the different responses.

The final step of the RSM is to acquire with Minitab 19 the dimensions and current density for the optimized model and calculate the optimized volume, pressure drop, flow rate and power consumption. These characteristics are inserted in a new finite element model in software Flux 2018.1, the
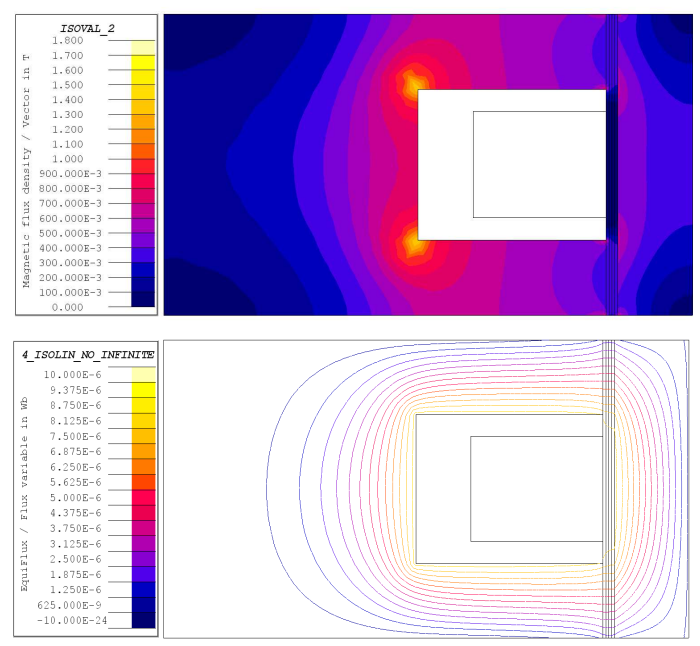

Fig. 8: Finite Element Model (FEM) simulation results for magnetic flux density and magnetic flux distribution in the 2D axis-symmetric cross-section of the optimal MR valve.

distribution of the magnetic field density is simulated, as in Fig. 8 and the magnetic field in the gap is calculated and presented in Fig. 9. It is obvious that the field gets its maximum values in the active regions of the valve, as expected.

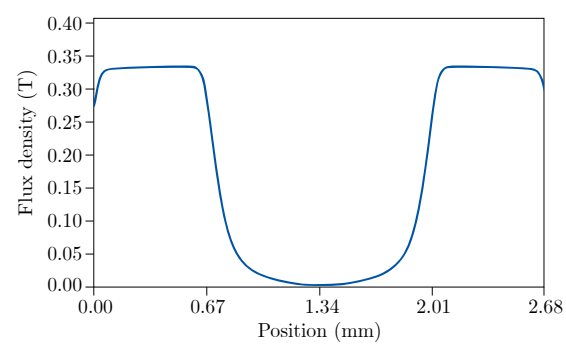

Fig. 9: Magnetic flux density distribution in the length of the gap, with maximum value in the active regions.

Table II demonstrates the values for the optimized model and compares them with the values of an existing, not optimized prototype. Indeed, starting with the pressure drop that marginally reaches the requirements of the system, it is indicated that there is still some space for improvement. However, the results show the achievement of the most important goal which is the miniaturization of the valve. We accomplished to obtain a total volume of $210.6 \mathrm{~mm}^{3}, 38 \%$ lower than the prototype's volume, with a much lower total height for the valve that will facilitate the miniaturization of the MR module and its integration in the smart insole. At the same time, there is a $50 \%$ decrease of the power consumption of each module, which is also crucial for our wearable system, so that we reduce the frequency of recharging.

Our future goal is to integrate the model of the pressure self-sensing phenomenon in the optimization of the MR module. The induced voltage characterization curve will 
TABLE II: Comparison between a valve prototype and the optimized model resulting from DoE

\begin{tabular}{|c|c|c|}
\hline Item & Prototype & Optimal Model \\
\hline Outer core radius $\mathrm{r}_{\mathrm{o}}(\mathrm{mm})$ & 5.0 & 5.0 \\
\hline Total valve's height $\mathrm{h}_{\mathrm{t}}(\mathrm{mm})$ & 4.3 & $\mathbf{2 . 6 8}$ \\
\hline Air gap g $(\mathrm{mm})$ & 0.1 & 0.108 \\
\hline Iron teeth's height $\mathrm{h}(\mathrm{mm})$ & 1.0 & $\mathbf{0 . 6 7}$ \\
\hline Wire diameter $\mathrm{D}_{\mathrm{w}}(\mathrm{mm})$ & 0.321 & 0.321 \\
\hline Fill factor $(\%)$ & 70.0 & 50.0 \\
\hline Current density $\mathrm{J}\left(\mathrm{A} / \mathrm{mm}^{2}\right)$ & 6.0 & 13.14 \\
\hline Total volume $\mathrm{V}\left(\mathrm{mm}^{3}\right)$ & 337.7 & $\mathbf{2 1 0 . 6}$ \\
\hline Flux density $\mathrm{B}_{\mathrm{g}}(\mathrm{T})$ & 0.527 & 0.341 \\
\hline Total pressure drop $\Delta P(\mathrm{kPa})$ & 1731.35 & 658.32 \\
\hline Flow rate $\mathrm{Q}\left(\mathrm{cm}^{3} / \mathrm{s}\right)$ & 3.14 & 2.44 \\
\hline Power consumption $\mathrm{P}_{\mathrm{w}}(\mathrm{mW})$ & 109.0 & $\mathbf{5 4 . 0 4}$ \\
\hline
\end{tabular}

be included as a factor of the DoE. It is, however, timeconsuming and costly to fabricate valves with all the different combinations of dimensions explained in the previous DoE process (16 different valves in total), in order to create the new experiment matrix. Thus, there is the intention to utilize the fractional factorial optimization technique to reduce the number of experimental runs and, consequently, the number of fabricated valves to 8 .

\section{CONCLUSIONS}

In the present work, we analyzed the design optimization of a MR valve. This valve is created for the first time with the aim to be deployed in a wearable insole solution that prevents diabetic patients from amputations. The objective of the optimization process was the minimization of the valve's volume and power consumption, as well as the maximization of pressure drop and flow rate. The factors affecting the optimum solution are the current density, the valve's height and air gap as well as the ratios between the inner core radius of the valve and outer core radius and the coil's width over the inner core radius. The final acquired optimal model greatly satisfied the requirements for volume and power consumption minimization. The pressure drop and flow rate were not maximum but they were still within the required range.

Besides the optimization procedure, a testbench was used in order to examine the pressure self-sensing possibilities of the MR valve. This idea arose from the existence of an induced voltage due to pressure variations. The next step will be the full characterization of the induced voltage and its correlation with pressure. It will, also, include the incorporation of these results in the DoE optimization process, investigating the induced voltage for different valves' configurations.

\section{ACKNOWLEDGMENT}

The authors gratefully acknowledge support from the BRIDGE funding programme, conducted by the Swiss National Science Foundation (SNSF) and Innosuisse-Swiss Innovation Agency.

\section{REFERENCES}

[1] M. R. Jolly, J. D. Carlson, and B. C. Munoz, "A model of the behaviour of magnetorheological materials," Smart Materials and Structures, vol. 5, no. 5, p. 607, 1996.

[2] A. Ghaffari, S. H. Hashemabadi, and M. Ashtiani, "A review on the simulation and modeling of magnetorheological fluids," Journal of intelligent material systems and structures, vol. 26, no. 8, pp. 881-904, 2015.

[3] J. De Vicente, D. J. Klingenberg, and R. Hidalgo-Alvarez, "Magnetorheological fluids: a review," Soft matter, vol. 7, no. 8, pp. 3701-3710, 2011.

[4] A. Milecki and M. Hauke, "Application of magnetorheological fluid in industrial shock absorbers," Mechanical Systems and Signal Processing, vol. 28, pp. 528-541, 2012

[5] F. Gordaninejad and S. P. Kelso, "Magneto-rheological fluid shock absorbers for HMMWV," Smart Structures and Materials 2000: Damping and Isolation, vol. 3989, pp. 266-273, International Society for Optics and Photonics, 2000.

[6] G. Savioz, V. Ruchet, and Y. Perriard, "Study of a miniature magnetorheological fluid actuator for haptic devices," IEEE/ASME International Conference on Advanced Intelligent Mechatronics, pp. 1197-1202, IEEE, 2010.

[7] X. Zhang, W. Li, and Y. Zhou, "A variable stiffness MR damper for vibration suppression," IEEE/ASME International Conference on Advanced Intelligent Mechatronics, pp. 106-111, IEEE, 2009.

[8] A. Y. Abd Fatah, S. A. Mazlan, T. Koga, H. Zamzuri, M. Zeinali, and F. Imaduddin, "A review of design and modeling of magnetorheological valve," International Journal of Modern Physics B, vol. 29, no. 04, p. 1530004, 2015.

[9] C.-W. Lung, E. T. Hsiao-Wecksler, S. Burns, F. Lin, and Y.-K. Jan, "Quantifying dynamic changes in plantar pressure gradient in diabetics with peripheral neuropathy," Frontiers in bioengineering and biotechnology, vol. 4, p. 54, 2016.

[10] B. J. Beuker, R. W. van Deursen, P. Price, E. A. Manning, J. G.Van Baal, and K. G. Harding, "Plantar pressure in off-loading devices used in diabetic ulcer treatment," Wound repair and regeneration, vol. 13, no. 6, pp. 537-542, 2005.

[11] D. Grivon, Y. Civet, Z. Pataky, and Y. Perriard, "Design and characterization of a soft magneto-rheological miniature shock absorber for a controllable variable stiffness sole," Archives of Electrical Engineering, vol. 64, no. 4, pp. 547-558, 2015.

[12] D. Grivon, Y. Civet, Z. Pataky, and Y. Perriard, "Detection of pressure or flow rate variations in MR valves through magnetic flux analysis," 2016 19th International Conference on Electrical Machines and Systems (ICEMS), pp. 1-5, IEEE, 2016.

[13] C. Chen and W.-H. Liao, "A self-powered, self-sensing magnetorheological damper," IEEE International Conference on Mechatronics and Automation, pp. 1364-1369, IEEE, 2010.

[14] G. Hu, Y. Lu, S. Sun, and W. Li, "Development of a self-sensing magnetorheological damper with magnets in-line coil mechanism," Sensors and Actuators A: Physical, vol. 255, pp. 71-78, 2017.

[15] H. Zhang, C. Liao, W. Chen, and S. Huang, "A magnetic design method of MR fluid dampers and FEM analysis on magnetic saturation," Journal of intelligent material systems and structures, vol. 17, no. 8-9, pp. 813-818, 2006.

[16] W.-M. Chen, T. Lee, P. V.-S. Lee, J. W. Lee, and S.-J. Lee, "Effects of internal stress concentrations in plantar soft-tissue-a preliminary three-dimensional finite element analysis," Medical engineering \& physics, vol. 32, no. 4, pp. 324-331, 2010.

[17] N. Hayafune, Y. Hayafune, and H. Jacob, "Pressure and force distribution characteristics under the normal foot during the push-off phase in gait," The foot, vol. 9, no. 2, pp. 88-92, 1999.

[18] R. F. Gunst and R. L. Mason, "Fractional factorial design," Wiley Interdisciplinary Reviews: Computational Statistics, vol. 1, no. 2, pp. 234-244, 2009.

[19] Wang, D. H., H. X. Ai, and W. H. Liao. "A magnetorheological valve with both annular and radial fluid flow resistance gaps," Smart materials and structures 18.11 (2009): 115001.

[20] R. Delivorias, "Application of er and MR fluid in an automotive crash energy absorber," Report no. MT04, vol. 18, 2004.

[21] A. Dean, D. Voss, and D. Dragulji, "Response surface methodology," Design and analysis of experiments, pp. 565-614, Springer, 2017.

[22] D. Grivon, "Design, modelling and sensing possibilities of magnetorheological based devices," tech. rep., pp. 106, EPFL, 2017. 